\title{
Le modèle POPOLE (POlitique de la POLlution des Eaux) Une tentative d'analyse du système "Eau" dans
une Agence Financière de Bassin"
}

\author{
par P.-F. Tenière-Buchot
}

Agence Financière de Bassin Seine-Normandie

Depuis 1966 la France est dotée d'organismes originaux, les Agences Financières de Bassin, qui gèrent le patrimoine eau tant sur le plan des ressources que sur celui de la préservation de la qualité.

Placées sous le contrôle de l'Administration centrale, les Agences tirent leurs ressources financières de redevances payées par les usagers de l'eau. Ces redevances (aux frais de fonctionnement près) sont reversées sous forme de subventions et de prêts à des usagers ou à des groupements d'usagers, de façon à facilliter la mobilisation et la gestion de la ressource en eau, ou pour intervenir dans la lutte contre la pollution des eaux.

Organismes d'exécution, les Agences préparent la politique hydraulique décidée par un Conseil d'Administration et une assemblée d'usagers (le Comité de Bassin) où sont présents des représentants de l'Administration (Environnement, Aménagement du territoire et Equipement, Agriculture, Industrie, Santé publique, Intérieur, Economie et Finances), des représentants élus des collectivités locales (maires, parlementaires), des représentants des usagers (industriels, pêcheurs à la ligne, association de riverains, batellerie).

Définissant les programmes d'intervention en cohérence avec la planification nationale, ces instances décident l'importance des redevances à percevoir et les modalités des interventions financières à effectuer. Des objectifs à long terme $(30$ ans) déterminés de manière collective permettent l'obtention d'une certaine continuité logique pour ces décisions à court-moyen terme (5 ans).

L'étude d'analyse de systèmes, qui est ici présentée, a eu pour objet de faciliter le choix d'objectifs à long terme en explorant systématiquement les divers futurs possibles en matière de ressources en eau et d'évolution de la pollution. Le système retenu était celui du bassin hydrogéographique Scine-Normandie $\left(100000 \mathrm{~km}^{2}, 15\right.$ millions d'habitants,
3 milliards de $\mathrm{m}^{3}$ d'eau prélevés par an, 2 millions de tonnes de pollution créée par an).

La méthode utilisée pour analyser ce système est l'analyse structurelle prévisionnelle. Approche globale faisant appel aux méthodes matricielles, à la théorie des graphes et à la recherche opérationnelle; elle se déroule en sept étapes:

1 - Recensement des variables intervenant dans le système.

92 variables groupées en offre en eau, demande en cau, contraintes externes de décor, contraintes internes politiques, ont été rassemblées. Ces variables sont aussi bien quantitatives que qualitatives, peuvent aussi bien concerner des individualités désignables que des caractères servant de critères de jugement subjectif.

\section{2 - Construction d'une matrice des relations logiques existant entre les variables.}

Reprises dans le même ordre en lignes et en colonnes, les variables déterminent une matrice dont les cases sont qualitativement remplies chaque fois qu'une influence d'une variable sur une autre est décelée.

L'observation des nuages de points ainsi obtenus, la répartition des points en lignes et en colonnes permet d'obtenir des hiérarchies significatives.

Une analyse plus fine peut être alors entreprise en distinguant les liaisons de causalité directe (conséquence automatique), les liaisons conditionnelles (existence d'un effet de seuil) et les liaisons décisionnelles (politique de l'expérimentateur).

* Le texte complet de cette étude a été publié par Analyse et Prévision-Futuribles, février et mars 1973. SEDEIS, 52, rue des Saints-Pères, 75007 Paris. 
3 -- Construction d'une matrice des relations fondamentales.

Ayant désigné des variables-objectifs au sein de la population des variables retenues, certaines variables appelées «variables motrices » sont mises en lumière à travers les liaisons indiquées dans la matrice des relations logiques.

Une sous-matrice composée des seules variables motrices et objectifs peut donc être construite. Elle est ordonnée suivant le degré décisionnel des variables. On distingue trois classes à cet égard:

- variables motrices décisionnelles (instruments politiques de l'expérimentateur);

- variables d'influence décisionnelle (zone d'influence de l'expérimentateur);

- autres variables motrices (contraintes et impondérables).

\section{4 - Synthèse des actions et orientations décisionnelles.}

C'est uniquement sur cette dernière classe que des prévisions seront effectuées. Elles complèteront les décisions volontaires prévisionnelles intéressant les objectifs et formeront les conditions à observer avant la prise de décision sur les variables des deux premières classes.
Les hypothèses de prévision et les décisions entrainées restent qualitatives à ce stade, en employant un symbolisme très simple d'acoroissement, de stagnation ou de diminution.

\section{5 - Construction d'un graphe associé au système.}

L'introduction des décisions prises, dans le sous-bloc des variables décisionnelles et d'influence décisionnelle de la matrice des relations fondamentales, doit permettre de déceler les incohérences éventuelles.

Pour ce faire, un graphe dual de la matrice fondamentale est construit. Par multiplication booléenne de la matrice, les boucles de réactions de ce graphe sont isolées et étudiées sous l'angle de leur convergence ou divergence. Cette opération permet une certaine simplification grâce à l'élimination des liaisons parasites.

\section{6 - Quantification du graphe.}

La mise sur ordinateur du graphe ainsi constitué n'est réalisable qu'après quantification des liaisons binaires représentées par chaque arc du graphe. Des expressions mathématiques ou des tableaux de correspondances sont introduits à ce stade, dans la mesure où des unités et des mesures ont pu être définies pour chaque variable. Dans le cas contraire,

\section{Tableau}

\section{Données}

Produit brut du Bassin......... milliards de francs

Densité de la population....... habitants $/ \mathrm{km}^{2}$

Capacité barrages-réservoirs.... millions de $\mathrm{m}^{3}$

Structure commerciale........ nombre de groupes

Max, aide au bon fonctionnement. pourcentage

Redevance pollution.......... francs/tonne/an

Modification budget......... millions de francs

Remboursements prêts......... millions de francs

Budget interventions pollution... millions de francs

\section{Chercheurs :}

- Substitution épuration...... hommes-années

- Débits. . . ........... hommes-années

- Entretien des stations....... hommes-années

- Refroidissement. . . ........ hommes-années

- Techniques d'épuration...... hommes-années

\section{Résultats}

Surface urbanisée......... $\mathrm{km}^{2}$

Coût investissement épuration... francs/tonne/an
Besoins domestiques......... milliards de $\mathrm{m}^{3} / \mathrm{an}$

Besoins des industries......... milliards de $m^{3} / a n$

Débit étiage Paris. . . . . . . . . $m^{3 / s}$

Substitution épuration........ millions de tonnes/an

Rendement technique épuration.. pourcentage

Débit des égouts publics....... $m^{3 / s}$

Energie à refroidir.......... milliards de $k W h / a n$

Aide au bon fonctionnement..... francs/tonne/an

Coút d'entretien des stations.... francs/tonne/an

Capacité stations épuration..... millions de tonnes/an

Epuration efficace............ millions de tonnes/an

Proportion stations aidées...... pourcentage

Polution rejetée............ millions de tonnes/an

Auto-épuration. . . ........ pourcentage

Echauffement des eaux......... pourcentage

Pollution résultante. ......... millions de tonnes/an

\section{Résultats financiers}

Recettes effectives.......... millions de francs

Dépenses. . ............. millions de francs

Recettes - dépenses. . . . . . . millions de francs 
des hypothèses doivent être formulées avec l'aide des hydrauliciens spécialistes.

\section{7 - L'exploitation sur ordinateur.}

Réduit au seul objectif «évolution de la pollution», le modèle Popole explore les modifications annuelles des variables suivantes durant la période 1970-2000 (voir tableau).

Des statistiques annuelles et pluriannuelles peuvent être obtenues. L'utilisation du mode conversationnel permet des simulations rapides des conséquences de modifications des variables d'entrée.

Les résultats de l'exploitation du modèle Popole sont multiples :

- Effet de l'infuence d'une variable d'entrée ou d'un groupe de variables d'entrée sur la pollution rejetée dans les rivières. Prévision des évolutions paramétrées en fonction des données.

- Recherche des conséquences de politiques financières différentes. Obtention de courbes d'indifférence entre plusieurs solutions envisageables. Détermination de taux marginaux de substitution.

- Recherche régressive des conditions nécessaires à la réalisation d'un objectif volontaire.

- Etude des effets de phénomènes connexes (échauffement des eaux, par exemple) sur l'objectif principal (état de pollution des rivières).

- Analyse du comportement des usagers face à certaines incitations financières dans le domaine de la lutte contre la pollution.

- Analyse des incohérences révélées par le modèle.

- Effets de perturbations techniques ou financières sur la stabilité du système.

- Détermination d'une programmation évolutive tenant compte des enseignements précédents.

Objet de réflexion et de sensibilisation (grâce à la possibilité de dialogue homme/machine), le programme Popole aura été un soutien important aux efforts de programmation de l'Agence de Bassin Seine-Normandie. Sa rusticité et son faible cô̂t auront certainement été les deux qualités permettant d'expliquer son efficacité.

\section{Discussion}

Président : M. C. Lefrou

M. le Président remercie M. Tenière-Buchot de la qualité de son exposé qui, malgré la technicité du sujet, a tenu en haleine tout lauditoire. Il ouvre ensuite la discussion.

M. LACRorx fait remarquer que le modèle décrit correspond à un jeu intellectuel très séduisant, mais dont il convient de contrôler très sérieusement les entrées et les sorties; en particulier, M. Lacrorx considère que l'indication obtenue par l'utilisation du modèle, d'après laquelle l'Agence n'était pas intéressée par la qua'ité de l'eau utilisée, ne peut avoir qu'une signification très provisoire; il sera nécessaire de revoir éventuellement les données introduites dans le modèle pour corriger cette conclusion.

J'en suis abso'ument persuadé, répond M. Tenièn-Buchot, mais cette indication quelque peu agressive n'avait d'autre objet que de montrer que dans l'état de pollution du Bassin ou du moins de certaines portions de celui-ci, le problème n'est plus de savoir faire de petits volumes d'eau potable de très bonne qualité - ce que l'on sait parfaitement faire - mais d'arriver à contrốter l'état de pollution de gros volumes d'eaux naturelles. Nous pensons que les efforts faits au cours de ces dernières années pour fabriquer une eau potable de qualité ont lasssé se dégrader le milieu naturel. Aussi, dans une optique d'environnement, nous avons fait bon marché du problème de l'eau potab'e parce que nous avons pensé qu'il y aurait toujours des personnes pour le traiter. Ce n'était donc pas tout à fait le rôle de l'Agence qui était de défendre l'environnement.

Vous auriez pu tout aussi bien admettre, observe $M$. le Président, que le maintien d'un certain nombre d'équilibres écologiques correspondait à des besoins nécessitant une certaine qualité de l'eau; dans ces conditions, vous auriez pu prendre comme variable principale pour l'Agence, non plus la gestion de la pollution, mais la fourniture d'une eau de qualité correcte à chacun des usagers; auriez-vous obtenu des résultats différents?

Très différents, répond $M$. Tenière-Buchot, qui renvoie au Livre blanc publié par l'Agence; celui-ci expose les détails d'une méthode qui n'a pu être présentée ici que sous une forme schématique, sinon caricaturale, en raison du peu de temps disponible.
M. G. de Marsily (Ecole des Mines, Fontainebleau) pose au conférencier la question suivante :

Vous avez signalé qu'il existait une valeur limite de la redevance pollution à une date donnée représentant, selon les lois du marché, la taxe maximum qu'acceptent de payer les usagers.

Le niveau de cette redevance est-il à l'heure actuelle en SeineNormandie, suffisant pour assurer les objectifs d'environnement que l'on peut raisonnablement se fixer?

\section{TENIÈRE-BUChot répond:}

Non, la redevance n'est pas encore suffisamment élevée. Nous pensons - et c'est ce que nous avions recommandé dans notre Livre blanc - que le triplement de sa valeur en 1970, c'est-ì-dire $6,60 \mathrm{~F}$ par habitant au lieu de $2,20 \mathrm{~F}$ (en francs 1970 ) correspondait à un niveau convenab's eu égard au montant des investissements, au coût de l'entretien des stations et également à la longueur du programme (30 ans) et aux facultés contributives des intéressés. En 1971 , la redevance a été doublée; elle est passée à $4,40 \mathrm{~F}$ par habitant, ce qui n'est pas encore suffisant et ne permet qu'un écrémage de la pollution. Pour des stations d'épuration comp'iquées, ce taux n'est pas encore très incitatif, mais nous espérons qu'à l'occasion du troisième programme de l'Agence, c'est-à-dire, vers 1976-1977, il y aura une réévaluation des redevances nous permettant de faire correspondre les recettes financières aux besoins qui ont été définis pour les trente prochaines années.

Avez-vous eu l'occasion d'examiner l'influence de la recherche sur l'évolution de la pollution dans les années à venir, demande M. BuYON (S.E.T.U.D.E.)?

Voici - sous toutes réserves - répond M. Tenière-Buchot, quelques-uns des résultats auxquels nous avons abouti.

Actuellement, il n'y a pas assez de travaux de recherches dans le domaine qui nous intéresse mais il n'en résulte pas immédiatement des conséquences trop graves.

A partir de 1975-1980, la recherche se présente comme l'instrumen de substitution des redevances; c'est-à-dire qu'il faut que des moyens 
soient mis en auvre en 1975-1980 pour prendre le relais des redevances en 1985

Pourquoi cela ? Parce que au fur et à mesure de la dépollution du Bassin, l'efficacité des redevances devient de moins en moins grande l'instrument financier s'émousse car le rendement technique des ouvrages d'épuration diminue. Nous avons des ouvrages d'épuration qui, dans le mellleur des cas, éliminent 80 à $83 \%$ de la pollution il reste 17 à $20 \%$ de la pollution initiale qui ne sont pas traités; mais ces 17 à $20 \%$ portent sur une masse de pollution produite de p'us en plus grande; finalement, le rendement des investissements à qualité constante, à technique constante, devient de moins en moins intéressant.

Si donc il n'y a pas de nouveaux appareils réalisant une épuration p'us poussée, - c'est là où nous allons retrouver la qualité des eaux dont parlait M. LACroIX, tout à Theure - nous allons faire du mauvais travail.

La recherche est alors indispensable comme facteur de substitution. On montre qu'il est nécessaire de changer sa politique, à partir de 1975-1980, car la recherche met au moins dix ans avant de déboucher sur que'que chose de concret.

Mais, au début du programme - et cela est tout à fait indiqué par les résultats obtenus sur notre modèle - c'est-à-dire dans les années 1970-1980, il n'apparaît pas indispensable de donner beaucoup d'argent à la recherche dans la mesure où le retard - du moins en Seine-Normandie - est tel que, même avec les anciennes stations d'épuration, on fait encore du bon travai!; inutile de raffiner, il suffit d'enlever au meilleur prix le plus de pollution possible.
Par contre, à un moment donné - à peu près at cours du VII Plan — il faudra changer de politique. L'efficacité de la recherche passe d'aileurs par un maximum pour une certaine dépense de recherche et diminue ensuite; cela résulte d'un phénomène d'encombrement; s'il y a trop de chercheurs, une partie de leur activité finit par être employée à organiser entre eux des congrès de moins en moins rentables.

Sur une question de M. le Président, M. Tenière-Bucrior précise, qu'à son avis, l'un des objectifs de la recherche devrait être d'améliorer le rendement et la fiabilité des stations d'épuration sans en augmenter le prix. D'ores et déjà, les installations de fabrication d'eau potable ont des rendements voisins de $100 \%$, mais leur coût actuel interdit d'en généraliser l'emp'oi pour remédier à la pollution des eaux naturelles.

M. Normand demande pourquoi, suivant le conférencier, la précision des relations fonctionnelles introduites dans le modèle influe peu sur les «tendances» mises en lumière par celui-ci.

En fait, répond M. TENIÉre-Buchot, on procède toujours à des calculs de sensibilité pour contrôler jusqu'à quel point il en est bien ainsi. Les courbes qui vous ont été présentées ont été «testées » à ce point de vue; on a constaté qu'elles ne se déformaient pas trop pour des variations de l'ordre de $50 \%$ des paramètres d'entrée.

En raison des exigences de l'horaire, $M$. le Président se voit dans l'obligation de clore la discussion avant qu'elle soit arrivée à son terme. Il donne la parole à M. WILCZYNSKI pour l'exposé de sa communication. 